\title{
Neuronal Activity of the Human Pretectal Area (Preliminary Report)
}

\author{
Hiroaki Sekino, Takanori Fukushima, Tomokatsu Hori, \\ Buichi Ishijma and Keiji SANo \\ Key words: Extracellular recording, Interstitial nucleus of Cajal, \\ Spasmodic torticollis, See-saw nystagmus, Stereo-encephalotomy. \\ Department of Neurosurgery, University of Tokyo, Japan.
}

Recently, the extracellular rocording of unit discharge in human cases is an usual technique, however, the data of unit activities in human extra-thalamic structures has not reported in literature. During stereotaxic operations for intractable pain, spasmodic torticollis and see-saw nystagmus, unit activities were recorded in the thalamus and the pretectal area including the interestitial nucleus of Cajal. In this paper, the authors will deal with unit recording in the pretectal area in man. The results of stimulation and destruction of the interstitial nucleus of Cajal in cases of spasmodic torticollis were reported elsewhere ${ }^{7) 8)}$.

In 11 operations of 2 cases of spasmodic torticollis, 1 case of see-saw nystagmus and 4 cases of intractable pain, the recording of unit discharges were performed. The coordinates of the target points for spasmodic torticollis and sec-saw nystagmus ${ }^{7 / 8)}$ were as follows: 7 to $9 \mathrm{~mm}$ posterior to the midpoint of the intercommissural line, 2 to $3 \mathrm{~mm}$ inferior to the intercommissural line and $2 \mathrm{~mm}$ lateral to the midsagittal plane. In cases of intractable pain, the coordinates of the taget points were 5 to $7 \mathrm{~mm}$ posterior to the midpoint of the intercommissural line, zero to $1 \mathrm{~mm}$ superior to the intercommissural line and 4 to $5 \mathrm{~mm}$ lateral to the lateral wall to the third ventricle. According to Schaltenbrand-Bailey's atlas, the former coordinates indicates the pretectal area including the interstitial nucleus of Cajal and the latter coordinates is the CM-Pf complex. The operations were performed under local anesthesia using $1.0 \%$ solution of procain. The head of the patient was freely movalble. Through a precentral burr hole, electrode was introduced to the target point. In all cases, the tracks of the electrodes passed through the two points represented by the coordinates mentioned above. Before insertion of the ordinary bipolar concentric electrode for stimulation and destruction, a microelectrode was put into the target point through a coated stainless steel tube, $1.0 \mathrm{~mm}$ in outer diameter. The guide tube was stereotaxically inseted and was fixed 5 to $10 \mathrm{~mm}$ above the target point. The microelectrode consisted of a 0.1 to $0.2 \mathrm{~mm}$ coated tungsten wire, the tip being $I$ to $2 \mu$ in diameter, which was held on a $0.8 \mathrm{~mm}$ coated stainless steel tube. The resistance of the microelectrode was 5 to $15 \mathrm{M} \Omega$. The cathode follower ampliphier, NIHON KODEN MZ-4, and the data recorder, TEAC R-401 were used for recording.

As shown in Table 1, 192 units were successfully recorded in the thalamus and in the pretectal area. The all needle tracks were shown in Fig. 1.35 units out of 192 were picked up in the pretectal area. When the microelectrode passed out the thalamus, the background activity changed and the amplitude of units was at- 
Ipsi. Rot.

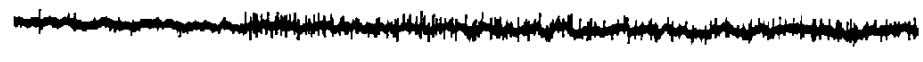

Contra. Rot.

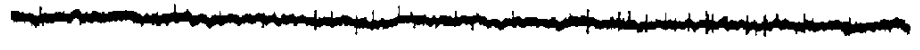

Post. flex

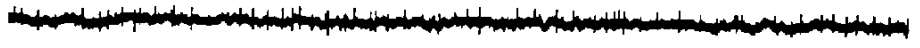

Ant. flex.

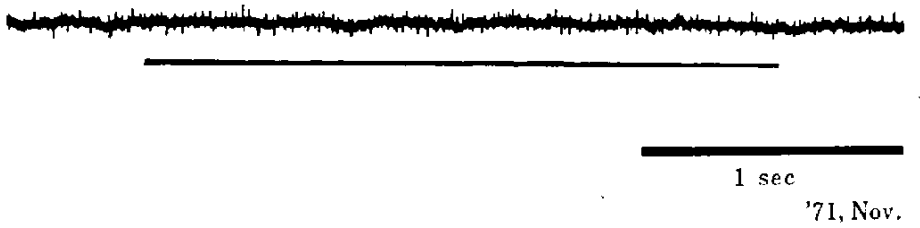

Fig. 2 Pretectal neuron and neck movement

duration of the voluntary head movement

to left

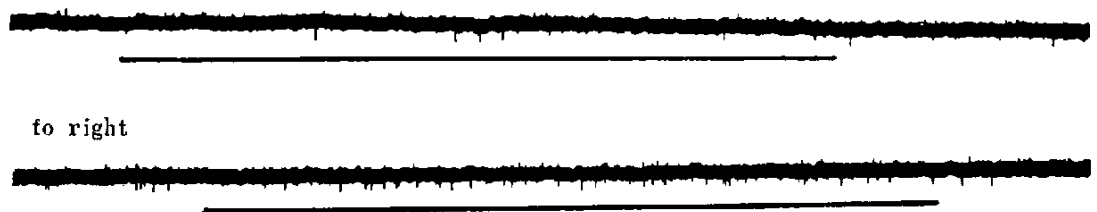

up

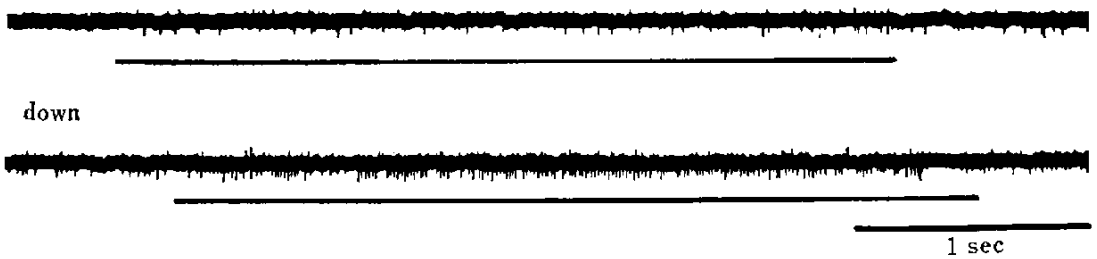

Fig. 3 Pretectal neuron and cyc movements duration of the voluntary eye movements.

tenuated. Then it was able to determine that the microelectrode was in the pretectal area. Sometimes, the site of the tip of the electrode was checked on X-ray films. After the units were stably recorded, the patient was asked to move his head of eyes.

Out of 35 units, 17 units had no response to the voluntary head or eye movements. 12 units were correlated with neck movements and 7 units increased their discharge with movements of eyes. The classification of the response patterns of those 12 neurons correlated with neck movements is shown on Table 2. Electromyelography of the bi- 
lateral sternocleidomastoid muscles and the bilateral trapezius muscles were recorded. Rotation of the head caused the EMG discharges of the sternocleidomastoid muscle on the side contralateral to the direction of head rotation and of the trapezius muscle on the ipsilateral side. The bilateral trapezius muscles contracted during posterior flexion of the head and the bilateral sternocleidomastoid muscles contracted during anterior flexion of the head. Increase of discharges of 6 units was induced by the head turning toward the contralateral side. In 2 units out of 6 , discharges increased during posterior flexion of the head. As the muscle which takes part with both rotation and posterior flextion of the head, is the trapezius muscle on the same side of rotation, these two units should be correlated with contraction of the contralateral trapezius muslcle or relaxation of the ipsilateral sternocleidomastoid muscle. At the ipsilateral turning of the head, 3 units increased in firing rate, as shown in Fig. 2. Units which had a relation with the flexion of the head were $3 ; 1$ unit in posterior flexion and 2 units in anterior flexion, respectively. Apparent suppression of unit discharges at the movement in the opposite direction was not obscrved, however, some ncurons seemed to decrease in number of discharges by rotation toward the opposite side.

7 units showed a responsc to eyc movements, as one of them was shown in Fig. 3.

As mentioned in our previous papers ${ }^{6 / 7) 8)}$, the authors have stimulated various points in the human brain stem. Upon stimulation, the subthalamus, the posteromedial hypothalamus, the red nucleus, the central tegmental tract and the inferior olive all caused lateral flexion of the head to the side ipsilateral to stimulation. Electrical stimula tion of the pretectal area including of the interstitial nucleus of Cajal in man elicited EMG discharges and contraction of the bilateral posterior neck muscles, dominantly on the side of stimulation accompanied by retroflexion of the head and sometimes accompanied by eye movements. As described in those prcvious papers, rotatory movement of the head and neck was not observed upon electrical stimulation of the human brain stem. Based on the studies of Hess ${ }^{2}$ on cats, Hassler ${ }^{1)}$ described that upon stimulation of the interstitial nucleus the rotatory movement of the head around the longitudinal axis toward the side of stimulation was elicited, the raising of the head occured by stimulation of the prestitial nucleus and the bending of the head was produced by stimulation of the nucleus of Darkshewitsch. Mabuchis) confirmed that stimulation of the area in and around the interstitial nucleus elicited rotatory movement of the head and eyes to the stimulation side and abration of this area caused the head rotation to the opposite side, in cats. In chronic implant human cases, Nashold4)5) said that the contralateral turning of the head and eyes was produced upon stimulation of the subthalamus and the dorsal midbrain as caudal as the inferior colliclus. According to the results of the present study, it is obvious that the neurons which have some relation with the movements of the head and neck, especially with rotatory movement, scatter in the pretectal area including the interstitial nucleus of Cajal. It seems that each neuron coresponds to each muscle. It is not clear whether these neurons arc centrifugal or centripetal.

In present study, number of units was not enough to further examination. Mechanism of neck movements and mechanism of spasmodic torticollis are very complicated and further studies will be necessary to elucidate these mechanisms. 


\section{Summary}

During stereotaxic operations for spasmodic torticollis, see-saw nystagmus and intractable pain, 192 units were recorded in the human thalamus and the pretectal area including the interstitial nucleus of Cajal. In this paper, the authors discussed the relation between the pretectal neurons and the movements of head and eyes. The pretectal neurons have some relation with the movements of the head and neck, especially with rotatory movement. It seems that each neuron coresponds to each neck muscle. It is not clear whether these neurons are centrifugal or contripetal.

\section{References}

1) Hassler, R.: In Field, Magoun and Hall. Handbook of Physiology Vol. II, pp. 890894, Americal Physiological Society, Washington D.C., 1960.

2) Hess, W. R.: Hypothalamus und Thalamus, Experimental-Dokumente, Thieme, Stuttgart, 1956.

3) Mabuchi, M.: Rotatory head response cvoked by stimulating and destracting the interstitial nucleus and surrounding region, Exp. neurol. 27, 175-193, 1970.

4) Nashold, B. S. and Gills, J. P.: Ocular signs from brain stimulation and lesions, Arch. Ophthal. 77, 609-618, 1967.

5) Nashold, B. S., Slaughter, G, and Gills, J. P.: Ocular reactions in man from deep cerebellar stimulation and lesions, Arch. Ophthal. 81, 538-543, 1969.

6) Sano, K., Yoshioka, M., Ogashiwa, M., Ohye, C., Sekino, H. and Mayanagi, Y.: Central mechanisms of neck movements in human brain stem, Conf. neurol.: 29, 107 $111,1967$.

7) Sano, K., Yoshioka, M., Mayanagi, Y., Sekino, H., Yoshimasu, N. and Tsukamoto, Y.: Stimulation and destruction of and around the interstitial nucleus of Cajal in man, Conf. neurol. 32, 118-125, 1970.

8) Sano, K., Sekino, H., Tsukamoto, Y., Yoshimasu, N. and Ishijima, B.: Stimulation and destruction of the region of the interstitial nucleus in cases of torticollis and see-saw nystagmus (in press). 